\title{
Appellationen am Großen Rat von Mecheln
}

\section{Einleitung: Die Doppeldeutig- keit des Begriffs "Appellation" im Zusammenhang mit der Praxis am Großen Rat und die Zuständigkeit für Appellationen}

Teilweise entspricht die am Großen Rat vom 15. bis zum 18. Jahrhundert verwendete Terminologie dem allgemeinen und noch heute üblichen Begriff des Appellationsverfahrens. Diese Feststellung ist nicht überraschend, da dieses Verfahren in den Niederlanden am Ende des Mittelalters wesentlich auf dem gelehrt-rechtlichen römisch-kanonischen Modell beruhte, ${ }^{1}$ daneben auch auf der französischen Praxis, ${ }^{2}$ besonders des Pariser Parlement. ${ }^{3}$ Allerdings ist

\footnotetext{
${ }^{1}$ In historisch-vergleichendem Rahmen noch immer grundlegend VAN CAENEGEM, History, für die (südlichen) Niederlande und den Großen Rat bes. 46-47, 50-52.

2 Der französische Einfluss soll am Ende des 15. und Anfang des 16. Jahrhunderts auch bei anderen Justizhöfen der alten Niederlande spürbar gewesen sein, siehe z.B. WEDEKIND, Bijdrage 11-23 (über Appellationen vom Hof von Holland zum Großen Rat im Allgemeinen 150-159).

3 Über Appellationen und das Appellationsrecht am Großen Rat liegt keine spezifische monographische Darstellung vor. Die beste Synthese für die Anfangsjahre bei VAN ROMPAEY, De Grote Raad, über Appellationen bes. 308-329 (Zuständigkeit) und 447-468 (Verfahren); teilweise auch aufgenommen in DERS., De procedure. Für erstinstanzliche Verfahren im 16. Jahrhundert: VAN RHEE, Litigation and Legislation. In der niederländischen Reihe Procesgidsen erschien bisher kein Band, der dem Großen Rat gewidmet wäre (siehe jedoch über den Hoge Raad, den nordniederländischen
}

die Ähnlichkeit auch nicht vollständig. In den meisten Rechtssystemen hat sich die Appellation aufgrund der Vorstellung eines grundsätzlichen Rechts einer Partei durchgesetzt, ihre Rechtssache bis auf einige Ausnahmen (wie z.B. Streitigkeiten eines niedrigen Wertes) sachlich durch ein neues (öfter: hierarchisch übergeordnetes) Richtergremium (oder wenigstens durch einen Richter, der eine höhere Stelle in der Gerichtshierarchie bekleidete) überprüfen zu lassen. Ursprünglich (d.h. im 15. und 16. Jahrhundert) und in der Theorie war das nicht ganz die Auffassung der Appellation, wie sie in den Niederlanden entwickelt wurde: ${ }^{4}$ Nach den dort herrschenden Modellen beschränkte sich ein Appellationsverfahren im engeren Sinne auf eine Überprüfung der Akten, die den Untersuchungs- und Entscheidungsprozess des Richters a quo, und natürlich dessen Schlussfolgerungen (insbes. seine Entscheidung) dokumentierten, ohne dass aber die Streitigkeit selbst einem neuen Untersuchungs- (und deshalb Beweis-)Verfahren unterworfen werden sollte. Auch das Fundament der Appellation könnte ursprünglich ein anderes gewesen sein: nicht „bottom up“, als ein Recht des Individuums als Rechtsuntertan oder Partei, sondern eher "top down", als die Manifestation der Gerichtsherrschaft des

\footnotetext{
„Nachfolger" des Großen Rates für Holland (ab 1582, später auch für Seeland): LE BAILly, VerHAS, De hoofdlijnen.

4 Über die Anfänge der Appellation in der Grafschaft Flandern: MONBALLYU, Van appellatiën ende reformatiën; am Rat von Brabant: GodDING, Le Conseil de Brabant 254-260.
} 
Fürsten (und dessen Aufgabe, seine Untertanen zu schützen). Jedoch sollten solche Unterschiede auch relativiert werden: In der Praxis ${ }^{5}$ beschäftigten sich viele Appellationsverfahren mit Untersuchungs- und Beweisfragen, und die gesellschaftliche Dynamik, die die Entwicklung der Appellationsprozesse vor den höheren fürstlichen Gerichten förderte, ging vor allem von den Parteien aus, nicht von den Generalprokuratoren oder anderen Vertretern der fürstlichen Gewalt.

In den Quellen zeigt sich auch eine andere Verwendung des Ausdrucks „Appellation“, die auf

${ }^{5}$ Die Rechtspraxis lässt sich rechtshistorisch zunächst aufgrund der Archivquellen des Großen Rates (im Brüsseler Allgemeinen Reichsarchiv) erforschen. Die mehr oder weniger ausführlich "beschreibenden“ Inventare der ehemaligen Arbeitsgruppe „Großer Rat von Mecheln“ (unter der Leitung von Prof. Dr. J. Th. de Smidt) sind am besten geeignet, um schnell Fragen im Zusammenhang mit Appellationsverfahren zu identifizieren; ansonsten sind die (zum großen Teil, besonders für die "flämischen“ Appellationen, noch nicht völlig inventarisierten) Prozessakten in Appellationsverfahren und die Urteile (sentences étendues) heranzuziehen, eine Arbeit, die bisher noch niemand unternommen hat. Das von der genannten Arbeitsgruppe behandelte Archivmaterial bezieht sich hauptsächlich 1. auf sämtliche Urteile des Großen Rates zwischen 1470 und 1580; 2. auf die Prozessakten in erstinstanzlichen und Appellationsverfahren, die eine Beziehung zu den nördlichen Niederlanden haben, und zwar für die Zeit von ca. 1460-1580. Für einen status quaestionis über den heutigen Stand der Inventare und der Forschung siehe meinen Rezensionsaufsatz WIJFFELS, Dutch litigation, mit bibliographischen Hinweisen auf u.a. die älteren Inventare. Eine weitere Quelle, die die Praxis dokumentiert und oft viele Auskünfte in appellationsrechtlichen Fragen bereithält, sind außerdem die Rechtsprechungssammlungen, die sich ganz oder teilweise auf den Großen Rat beziehen: WIJFFELS, Legal Records and Reports; DERS., Grand Conseil de Malines: La rédaction, und DERS., Grand Conseil de Malines: recueil. Auch die altbelgische Konsilienliteratur (in der aber nicht immer erwähnt wird, vor welchem Gericht die Rechtssache verhandelt wurde) bietet viel Material zum Thema Appellation(srecht): WAGNER, Niederlande, bes. 14171430. ein Verständnis des Appellationsbegriffs in einem weiteren Sinn hinweist. "Appellation", „appellieren“ (in den Gerichtssprachen des GroBen Rates, Französisch oder Niederländisch, manchmal auch Latein ${ }^{6}$ ) wird gelegentlich auch für andere Rechtsmittel als die Appellation im engeren Sinne verwendet, außerdem um verschiedene Verfahren zu bezeichnen, durch welche eine nicht-justizielle, sondern (modern ausgedrückt) (quasi-)behördliche Entscheidung vor dem Großen Rat angefochten wurde.

Um diese unterschiedlichen Bedeutungen der Appellation richtig $\mathrm{zu}$ verstehen, muss man sich die Entstehungsgeschichte und die Organisation des Großen Rates vergegenwärtigen. Der Große Rat wurde nicht $\mathrm{zu}$ einem bestimmten Zeitpunkt gegründet, sondern entwickelte sich gemäß einem Verlauf, der in mehreren Ländern im Spätmittelalter beobachtet werden kann, zunächst als ein spezialisiertes Gremium, das sich innerhalb des allgemein zuständigen fürstlichen Rates (in diesem Fall der Curia Ducalis der Herzöge von Burgund) allmählich mehr und mehr speziell mit Rechtsstreitigkeiten befasste. ${ }^{7}$ Unter der Bezeichnung "Großer Rat" entstand dann (während der Jahre 1435-1445) eine vom Herzogsrat getrennte Institution, deren Hauptaufgabe - damit auch Zuständigkeit - war, die Justiz im Namen des Herzogs auszuüben. Im

\footnotetext{
${ }^{6}$ Dies betrifft sowohl die Prozessakten als auch die Urteile. Die mir bekannte Gesetzgebung (aus der burgundischen und habsburgischen Zeit) ist immer auf Französisch (anders allerdings für Gerichte in einer hauptsächlich niederländisch-sprachigen professionellen Umwelt wie die Räte von Brabant und Flandern). Alte Drucke, in denen die Gesetzgebung über den Großen Rat gesammelt wurde, sind ebenfalls auf Französisch, z.B. Ordonnances, mit vielen Hinweise im Sachregister am Ende (s.v. „Appel“). ${ }^{7}$ Ursprung und Frühgeschichte des Großen Rates wurden grundlegend erforscht durch VAN ROMPAEY, De Grote Raad. Siehe auch MAEs, Het Parlement en de Grote Raad. Für eine Kurzübersicht über die gesamte Geschichte des Rates siehe GILISSEN, De Grote Raad; WIJFFELS, Grote Raad.
} 
15. Jahrhundert wurde der Große Rat für kurze Zeit (1473-1477) durch Herzog Karl den Kühnen teils nach französischem Muster als ein Parlement mit Sitz in Mecheln eingerichtet. Erst ab 1503/04, als sich die habsburgische Herrschaft in den Niederlanden dauerhaft behaupten konnte, wurde der Rat wieder mit festem Sitz in Mecheln installiert, wo er bis zum Anschluss der österreichischen Niederlande durch die revolutionäre französische Republik tätig war, sieht man einmal von einigen Unterbrechungen anlässlich politischer Unruhen oder Kriegswirren ab. Die Verordnungen des späten 15. und des 16. Jahrhunderts, durch die die Organisation und das Funktionieren des Rates geregelt wurden, zeigen, wie der Rat allmählich immer mehr hauptsächlich als ein Justizhof betrachtet wurde. Seine Höchstgerichtsbarkeit übte der Rat sowohl erstinstanzlich (in sog. privilegierten Rechtssachen $)^{8}$ wie als Appellationshof aus. In einem Zeitalter, das "Policey" und "Justiz" nie ganz trennte, behielt der Rat außerdem immer einige Zuständigkeiten im Bereich der fürstlichen Verwaltung. Als Justizhof war er nicht nur erstinstanzliches Gericht und Appellationshof, denn er konnte auch aufgrund anderer Rechtsmittel als der Appellation (z.B. Evokation, Revision ${ }^{9}$ usw.) zuständig sein. Auch in seinen anfänglichen Bestrebungen, sich als oberster Appellationshof in den burgundischen bzw. habsburgischen Niederlanden zu etablieren, konnte sich der Große Rat nicht ganz durchsetzen: Erstens gelang es schon spätestens Anfang des 16. Jahrhunderts einigen Provinzialgerichten (Brabant, Hennegau) endgültig, als „Souverängerichtshö$\mathrm{fe}^{\text {" }}$ anerkannt $\mathrm{zu}$ werden, und so zu vermeiden, dass ihre Entscheidungen durch Appellation am Großen Rat angefochten werden konnten. ${ }^{10}$

\footnotetext{
${ }^{8}$ VAN RHEE, Litigation and Legislation 27-52.

${ }^{9}$ KOSTER-VAN DIJK, WiJfFELS, Les procédures en révision.

${ }^{10}$ Am Ende des Ancien Régime beschränkte sich die territoriale Zuständigkeit des Großen Rates auf das
}

Zweitens entwickelte auch der Geheime Rat, ${ }^{11}$ ebenfalls seit dem Beginn des 16. Jahrhunderts, eine außerordentliche Zuständigkeit in Justizsachen, wodurch er erstinstanzlich oder durch privilegierte Rechtsmittel eine erhebliche Justiztätigkeit entfaltete, die es manchmal ermöglichte, eine Entscheidung des Großen Rates auf dieser „höheren" Ebene weiter anzugreifen.12 Wie in vielen anderen europäischen Ländern der Neuzeit, war deshalb auch die Höchstgerichtsbarkeit des Großen Rates nur relativ. ${ }^{13}$

kleine Territorium von Mecheln sowie die Grafschaften Flandern und Namur. Zu dieser Entwicklung siehe GILISSEN, De Grote Raad, und MAEs, Het Parlement en de Grote Raad, wo die Entwicklung der territorialen Zuständigkeit auch anhand von Karten illustriert wird.

${ }^{11}$ Das Rechtsmittelverfahren am Geheimen Rat darf wohl nicht nur als eine höhere Instanz im Verhältnis zum Großen Rat betrachtet werden; in manchen Rechtsstreitigkeiten war das Verhältnis viel komplexer, siehe z.B. SAINCT VAAST, DU LAURY, La jurisprudence (eigentlich eine erweiterte Fassung der erfolgreichen und schon im 17. Jahrhundert verbreiteten handschriftlichen Sammlung von Nicolas du Fief), Arrêt 76, 134-135: „Un Conseil Provincial, étant chargé par le Conseil Privé, de ne prononcer Sentence en quelque cause, sans lui en communiquer la resolution, communication étant faite, et ledit Conseil Privé aiant ordonné, ce que devoit être prononcé, il y échoit apel de telle Sentence“. In diesem Fall konnten sich die Richter am Provinzialhof von Flandern nicht auf eine Entscheidung einigen, woraufhin der Geheime Rat nach Untersuchung der Sache dem Provinzialhof auftrug, das Urteil in einem bestimmten Sinn zu sprechen. Später wollte eine Partei gegen dieses Urteil an den Großen Rat appellieren, was anlässlich eines „Zwischenfalles“ (incident) durch den Geheimen Rat genehmigt wurde; schließlich (am 4. 6. 1644) sprach der Große Rat ein Urteil, der Antrag auf Evokation an den Geheimen Rat wurde abgelehnt (22. 6. 1644).

12 SCHEPPER, De Geheime Raad; DERS., De Grote Raad; DERS., Rechter en Administratie.

${ }^{13}$ WijfFeLS, The Great Council. In diesem Sinne lässt sich die Lage, wenn auch wohl aufgrund anderer politischer Umstände, mit der territorial zersplitterten Höchstgerichtsbarkeit im Alten Reich (durch die Wirkung der Privilegien de non appellando), in Frankreich (durch die Gründung der sog. Provinzial-Parlements und der Souveränjustizhöfe seit dem 15. Jahrhundert), 
$\mathrm{Zu}$ diesem knappen Hinweis auf die Stellung des Großen Rates in der komplexen Gerichtsstruktur der alten Niederlande soll noch hinzugefügt werden, dass die Appellationsverfahren meistens entweder in erster oder zweiter Appellationsinstanz (im engeren Sinne) durchgeführt wurden, denn im Fall einer Appellation gegen die Entscheidung eines Provinzialrates konnte diese Entscheidung entweder in einem erstinstanzlichen Prozess vor dem Provinzialrat, oder nach einem Appellationsverfahren gegen die Entscheidung eines Lokalgerichts (z.B. eines Stadtgerichts) ergangen sein. Daneben gab es auch Fälle, in denen der Große Rat eine unmittelbare Appellationsgerichtsbarkeit gegenüber einem nicht-fürstlichen (erstinstanzlichen oder eben Appellations-)Gericht ausübte. ${ }^{14}$ Solche Unterschiede im Instanzenzug prägten, wie im Folgenden noch darzulegen ist, die wesentlichen Züge des Appellationsverfahrens.

$\mathrm{Zu}$ den Fragen, die sich in bezug auf die $\mathrm{Zu}-$ ständigkeit des Großen Rates als Appellationsgericht wiederholt stellten, gehörte jene der Appellationen omisso medio, wobei vor allem die Fälle in Betracht kamen, in denen der ordentliche Instanzenzug eine Berufung von einem Schöffengericht an einen Provinzialhof vorsah, der Berufungskläger aber versuchte, seine Appellation unmittelbar am Großen Rat einzulegen (und den Provinzialhof damit überging). Solche Appellationen, die ein politisch heikles Thema sowohl bei den Stadtbe-

in Italien (wegen der Vielzahl der Rote, Senati als grandi tribunali auf der Halbinsel) und in einigen anderen Ländern vergleichen.

${ }^{14}$ Beispiele sind die sog. Terres de débats (einige Dörfer im Grenzgebiet zwischen den Grafschaften Flandern und Hennegau, die von beiden Territorialobrigkeiten beansprucht wurden). Auch gegen Urteile des Stadtgerichts von Valenciennes wurde direkt an den GroBen Rat appelliert; in Seeland sollten nach einigen Quellen die Parteien die Wahl haben, gegen Urteile der Lokalgerichte entweder zunächst an den Hof von Holland zu appellieren (mit der Möglichkeit einer weiteren Appellation nach Mecheln), oder das Urteil in Form einer "Reformation“ direkt vor dem Großen Rat anzufechten. hörden wie bei den Provinzialhöfen waren, wurden grundsätzlich verboten, und der GroBe Rat sollte den betreffenden Berufungskläger an den Provinzialrat zurückverweisen. ${ }^{15}$ Trotzdem war bei manchem Appellanten die Versuchung offensichtlich groß, eine Appellationsinstanz zu überspringen.

Die Praxis zeigt, dass der Große Rat tatsächlich entsprechende Zurückverweisungen aussprach. Trotzdem waren solche Appellationen in unterschiedlichen Situationen möglich, wie zwei Beispiele aus den 70er Jahren des 16. Jahrhunderts belegen. In einem Streit zwischen Privatparteien über Getreidezinse wurde die Berufung gegen das Urteil des lokalen Gerichts im kleinen Ort Ozo (Herzogtum Luxemburg) am Provinzialhof von Luxemburg mit Zustimmung des Beklagten rückgängig gemacht, und der Große Rat erlaubte dem Berufungskläger, seine Appellation vor dem Großen Rat selbst fortzusetzen (das Urteil des Rates vom 8. März 1572 änderte die Entscheidung des Gerichts von Ozo ab und verurteilte den Appellanten zu einer niedrigeren Zahlung). ${ }^{16}$ Das zweite Beispiel betrifft eine familiäre Auseinandersetzung in Brügge zwischen Parteien spanischer Herkunft; Gegenstand des Streites war die Erziehung eines zwölfjährigen Mädchens, das einige Verwandte nach Burgos mitnehmen wollten. Dieser Umzug wurde durch eine Entscheidung des Brügger Gerichts untersagt. Die Berufung gegen dieses Urteil wurde im Auftrag des Landvogts omisso medio vor dem Großen Rat (statt dem Provinzialrat von Flandern) durchgeführt; der Rat machte das Urteil von Brügge rückgängig und ordnete andere Maßnahmen zum Schutz des Mädchens an (1576). ${ }^{17}$ Im ersten Beispiel wurde eine Appellation gewissermaßen omisso medio offensichtlich aufgrund eines Einverständnisses zwischen den Parteien, und zwar mit Geneh-

\footnotetext{
${ }^{15} \mathrm{Zu}$ dieser Regelung und der Praxis in den Anfangsjahren des Rates VAN ROMPAEY, De Grote Raad 320324.

${ }^{16}$ Brüssel, Algemeen Rijksarchief, Grote Raad van Mechelen, Geëxtendeerde Sententiën 872.96 (17591781), DE SMIDT, Chronologische lijsten Nr. 4080 (8. 3. 1572 st.n.).

${ }^{17}$ Brüssel, Algemeen Rijksarchief, Grote Raad van Mechelen, Geëxtendeerde Sententiën 877.6 (65-144), DE SMIDT, Chronologische Lijsten Nr. 4365 (6. 2. 1576 st.n.).
} 
migung (und deshalb unter der Kontrolle) des Großen Rates zugelassen, im zweiten Beispiel war eine Intervention der fürstlichen Gewalt (vielleicht auf Antrag einer Prozesspartei) erforderlich (ein Verfahren, das z.T. mit einer Evokation verglichen werden kann). Im Allgemeinen scheinen Kontroversen anlässlich von Versuchen, omisso medio zu appellieren, im Laufe des 16. Jahrhunderts aber eher selten vorgekommen zu sein. ${ }^{18}$

Bei der Beurteilung der historischen Quellen der Appellationsprozesse am Großen Rat ist diese Zweideutigkeit daher zu berücksichtigen: Einerseits findet man viele Rechtssachen, die dem heutigen Begriff der Appellation ziemlich genau entsprechen, andererseits wurde der Terminus "Appellation" auch in einem breiteren und offenen Sinne verwendet, so dass sich die Regeln, die die Appellation und andere Rechtsmittel prägten, gegenseitig beeinflussten und teilweise überschnitten. ${ }^{19}$

\section{Historische und juristische Quellen}

Die wichtigsten primären Quellen, die für eine Untersuchung der Appellation verfügbar sind, können (etwas künstlich) in historische und juristische Quellen eingeteilt werden.

\footnotetext{
18 Trotzdem sind den Appellationen omisso medio mehrere Abschnitte in den Decisiones von Paulus Christinaeus gewidmet: CHRISTINAEUS, Practicarum quaestionum [...] Decisiones.

${ }^{19}$ Siehe z.B. meine case-study: WijfFELS, Ius commune and International Wine Trade, und DERS., A Consultancy on Wine Imports. In diesem Fall wurde ein Rechtsmittel (wohl eine sog. Opposition) gegen eine Beschlagnahme durch die zuständige Stelle in Middelburg vor dem Großen Rat eingelegt. Sachlich wurde dieser Prozess als erstinstanzliches Verfahren angesehen, teilweise sind die Akten dennoch im Archiv des Großen Rates unter den „Appellationen von Holland" abgelegt worden.
}

\subsection{Juristische Quellen}

\subsubsection{Normative Rechtsquellen: Gesetzgebung und stylus}

Mehrere fürstliche Verordnungen aus der spätburgundischen Zeit und aus der ersten Hälfte des 16. Jahrhunderts enthalten eine ausführliche Reglementierung der Organisation und des Verfahrens am Großen Rat. ${ }^{20}$ Die Reihe endet mit der Ordnung von 1559, die bis zum Ende des Ancien Régime in Kraft blieb. Nach 1559 waren meistens nur noch Einzelaspekte des Verfahrensrechts Gegenstand der Gesetzgebung oder gesetzlicher "Erklärungen“. ${ }^{21}$ Die wichtigsten Verordnungen aus dem letzten Drittel des 15. Jahrhunderts und aus dem 16. Jahrhundert enthielten auch einen besonderen Abschnitt über das Appellationsverfahren am Großen Rat. Wie immer bei solchen Verordnungen stellt sich die Frage, in welchem Maße der Gesetzgeber die Hauptschritte eines „Musterverfahrens" beschreiben wollte, oder aber versuchte, in der Praxis entstandene Kontroversen zu klären, und deshalb, in wie weit die Verordnungen den normalen oder üblichen Fortgang eines Verfahrens darstellen, oder aber problematische Stufen des Prozessrechts widerspiegeln. Eine weitere Frage ist, in welchem Maß die angeordneten Regeln v.a. dem gelehrten Recht als „Idealnormativität" entnommen sind, ${ }^{22}$ oder aber als Auf-

\footnotetext{
${ }^{20}$ Die Verordnungen für die burgundische Zeit gedruckt bei VAN ROMPAEY, De Grote Raad, insbes. eine Verordnung ohne Datum (um 1470), 482-491, sowie die Verordnung von (ca.) 1.-8. 12. 1473, 493-506; für das 16. Jahrhundert siehe insbes. die Ordonnance de l'Empereur, portant règlement pour le Grand Conseil de Malines (April 1522), LAURENT, LAMEERE, Recueil des ordonnances II/2, 175-187, und die Prozessordnung (in der Edition unter dem Titel Style et manière de procéder du Grand Conseil de Malines) vom 8. 8. 1559, ebd. II/7, 461-492.

${ }^{21}$ Teilweise aufgenommen in: Ordonnances.

${ }^{22}$ Immerhin hatte das gelehrte Verfahrensrecht auch eine sehr konkrete Bedeutung in der Alltagspraxis: Die Prokuratoren und Anwälte beriefen sich häufig
} 
zeichnung des in der alltäglichen Praxis angewandten stylus curiae aufzufassen sind. Allerdings wird allgemein nicht angenommen, dass der Verfahrensstil des Großen Rates in den größeren Prozessordnungen, auch nicht in jener von 1559, systematisch „kodifiziert“ worden sei.

Als Beispiel kann die schon im spätmittelalterlichen gelehrten Prozessrecht bekannte und oft diskutierte Frage der Appellation gegen ein Zwischenurteil gelten. Einerseits gehörte die Möglichkeit, gegen solche Urteile Berufung einzulegen, zu den konsequenten Grundsätzen des kontradiktorischen Verfahrens, andererseits brachte gerade eine solche Appellation das Risiko mit sich, missbraucht $\mathrm{zu}$ werden oder einfach - selbst bona fide - eine Verlängerung der Prozessdauer und höhere Prozesskosten herbeizuführen. Auch in der Praxis des Großen Rates kamen von Anfang an solche Appellationen vor, und sie wurden regelmäßig durch den Berufungsbeklagten bekämpft. Aus den Akten geht hervor, dass ein wichtiges Argument gegen diese Berufungen das Prinzip war, dass eine Appellation gegen ein Zwischenurteil insofern nicht zulässig sei, als die Folgen dieser Entscheidung im Endurteil noch "reparabel“, d.h. zu vermeiden waren. Mit diesem Grundsatz wurden aber nicht alle Kontroversen beseitigt, denn was nun z.B. als „reparabel“ oder „nicht reparabel“ betrachtet werden sollte, konnte durch die Parteien je nach Interesse unterschiedlich bewertet werden. ${ }^{23}$ Auch in solchen Fällen behielt der Rat einen Beurteilungsspielraum. Dies wurde schließlich indirekt durch den Gesetzgeber auch zugestanden: Die Ordnung von 1559 bestimmte, dass Appellationen gegen Zwischenurteile nur nach den Bestimmungen des gelehrten (oder römischen) Rechts (,voulons estre gardée et observée la disposition du droit civil") zugelassen seien. ${ }^{24}$ Die Prüfung, ob die Bedin-

auf die Texte der corpora iuris und auf das gelehrte juristische Schrifttum, wenn sie einzelne Argumente über verfahrensrechtliche Fragen vorbrachten: WIJFFELS, Qui millies allegatur.

${ }^{23}$ Auch die Justizhöfe haben über diese Frage bisweilen gestritten, siehe z.B. GOOVAERTS, Inventaires 1, Nr. 955.

${ }^{24}$ Ordnung Style et manière de procéder (8. 8. 1559), TERLINDEN, BOLsÉE, Recueil des ordonnances II/7, 461492, V.13. gungen des gelehrten Prozessrechts im konkreten Fall erfüllt waren, sollte dadurch erleichtert werden, dass den Parteien die Vorlage des Zwischenurteils (oder mindestens des Urteilstenors) am Großen Rat auferlegt wurde. Das gelehrte juristische Schrifttum bot jedoch für solche Fälle viele Anhaltspunkte für Ausnahmen oder Unterscheidungen, die durch Praktiker ausgenützt wurden..$^{25}$

\subsubsection{Juristisches Schrifttum}

Nur sehr wenige Werke wurden spezifisch dem Verfahrensrecht am Großen Rat gewidmet. Eine der bekanntesten Ausnahmen (allerdings ein Werk, das sich nicht ausschließlich mit dem Großen Rat beschäftigt) ist die „Praktijke civile" von Philip Wielant, ${ }^{26}$ die auch die Grundlage des europäischen juristischen Bestsellers der Frühen Neuzeit, der „Praxis rerum civilium“ von Joost de Damhouder, ${ }^{27}$ war. Sonst kann man nur wenige Werke nennen, selbst im unveröffentlichten Schrifttum, die den stylus des Rates im Allgemeinen oder verfahrensrechtliche Sonderfragen in bezug auf die Praxis des Rates behandeln. ${ }^{28}$

Besser vertreten ist die praxisorientierte Literatur der Rechtsprechungs- und Konsiliensammlungen. In jenen Rechtsprechungssammlungen, die überwiegend oder wenigstens $\mathrm{zu}$ einem erheblichen Teil über Entscheidungen des GroBen Rates berichten, spielen auch verfahrens-

\footnotetext{
${ }^{25}$ Auch zu den Kontroversen über die Appellation gegen Interlokute enthalten die Decisiones von Paulus Christinaeus mehrere Abschnitte, in denen die Grundsätze und Quellen des gelehrten Rechts eine bedeutende Rolle spielen: CHRISTINAEUS, Practicarum quaestionum [...] Decisiones.

${ }^{26}$ WIElant, Practijke civile. Philip Wielant (14411520) machte ab 1471 Karriere als Rat am burgundischen Rat (und am Parlement von Mecheln) und war ab 1504 Vizepräsident des Großen Rates, siehe die biographische Notiz von DAUCHY, Wielant.

${ }^{27}$ DE DAMHOUDER, Practycke in civile saecken.

${ }^{28}$ Außerdem gab es für den Großen Rat wohl privat oder intern-amtlich angelegte Formularbücher, die aber nur wenige Erläuterungen über den stylus enthalten, siehe z.B. DE SMIDT, Quelques remarques; VAN DER MADE, Formulaires du XVIe siècle.
} 
rechtliche Fragen eine bedeutende Rolle. Das wichtigste Beispiel sind die "Decisiones" von Paulus Christinaeus, dessen Sammlung in den Bänden 2 bis 4 der Ordnung der Titel des Codex Justinianus folgt, so dass die den Titeln C. 7.6269 entsprechenden Absätze spezifisch Appellationsfragen zum Gegenstand haben. ${ }^{29}$ Die Konsilienliteratur hat sich in den südlichen Niederlanden verhältnismäßig bescheiden entwickelt, und die Veröffentlichungen lassen nicht immer erkennen, vor welchem Gericht ein Gutachten unterbreitet wurde. ${ }^{30}$ Sowohl die Decisiones wie die Consilia waren überdies eher durch das gelehrte Recht, in bestimmten Bereichen durch die aufgeschriebenen (und ggf. offiziell anerkannten) Gewohnheiten ${ }^{31}$ (zu denen der stylus der Gerichte nicht gerechnet wurde) und die (v.a. fürstliche) Gesetzgebung geprägt. Deshalb wird nur selten auf die spezifische Praxis eines Justizhofes anlässlich von Verfahrensfragen einge-

\footnotetext{
${ }^{29}$ Christinaeus, Practicarum quaestionum [...] Decisiones. Weil der erste Band nicht der Ordnung der Codex-Rubriken folgt, müssen dort die einzelnen Abschnitte (Decisiones) gesichtet werden; der Index rerum ermöglicht es, die wichtigsten Texte über das Appellationsrecht aufgrund der summaria zu identifizieren. WiJfFels, Van Paul van Christijnen; über den Aufbau und die Quellen von Christinaeus DERS., Legal Records and Reports 193-196.

${ }^{30}$ Eine der ersten veröffentlichten Konsiliensammlungen, die recht viel über Appellationen enthält (obwohl meistens nicht spezifisch am Großen Rat), ist die von EvERARDUS, Consilia. Everardus war Rat am, später Vorsitzender des Großen Rates. Eine der wichtigsten Konsiliensammlungen aus Belgien ist die von WAMESIUS, Responsorvm sive consiliorvm ad ivs, sowie DERS., Responsorum sive consiliorum de iure pontificio. Der Löwener Rechtslehrer Wamesius starb 1590, seine Konsilien wurden aber erst im Laufe des 17. Jahrhunderts gedruckt. Wamesius ist auch der Verfasser eines Traktats über Appellationsrecht: DERS., Tractatus de appellationibus (anders als der Titel vermuten lässt, handelt es sich v.a. um eine rechtsdogmatische Darstellung des gelehrten Kirchenrechts über Appellationsverfahren, insbes. einen Kommentar zu X. 2.28).

${ }^{31}$ WiJfFELS, La coutume.
}

gangen, ${ }^{32}$ was aber z.T. von den Interessen des Autors des Sammlung abhängt.

$\mathrm{Zu}$ den juristischen Quellen sind auch die Grundquellen des gelehrten Rechts (die Texte der corpora iuris) zu rechnen. Diese Quellen (und das gelehrte juristische Schrifttum, das sich als Kommentare unmittelbar auf sie bezog) wurden, wie schon erwähnt, z.T. indirekt oder implizit in die Prozessordnungen und den stylus curiae aufgenommen. Ausnahmsweise konnte die Begründung der Gesetzgebung auch ausdrücklich sein: So erwähnte die Ordnung von 1559, dass der Appellationstermin von 10 Tagen dem "geschriebenen Recht" entspreche. ${ }^{33}$ Freilich hatte das gelehrte Prozessrecht nur eine allgemeine, subsidiäre Bedeutung für das Partikularprozessrecht eines bestimmten Gerichts; in den Schriftsätzen der Anwälte (seltener der Prokuratoren) erweist sich jedoch, dass die gelehrtrechtlichen Quellen zum Prozessrecht (u.a. auch zum Appellationsverfahren) eine bedeutende Rolle spielten, wenn im Laufe des Prozesses

\footnotetext{
${ }^{32}$ Mir ist keine monographische Literatur bekannt, die speziell dem stylus des Großen Rates gewidmet ist. Unterschiedlich ist die Lage für andere Justizhöfe in den südlichen Niederlanden, z.B. den Rat von Brabant, siehe DEKKERS, Bibliotheca Belgica, s.v. Branlet (J.J.), Dieven (W.A. van), Loovens (J.E.). Das Werk von VAN DER TANERIJEN, Boec, entspricht wohl nur teilweise der Praxis des Brabanter Rates, immerhin sei hingewiesen auf die Besonderheiten der Appellation vor dem Rat, dargestellt in Teil II, Abschnitt 19, 128136. Das Werk von WiELANT, Practijke civile, wird oft als eine Darstellung des stylus des Großen Rates und des Rates von Flandern angesehen, ist aber durch gelehrt-rechtliche Konzepte und Zusätze geprägt. Das Buch von CUYPERS, Tractaet, betrifft zunächst die Praxis am Lokalgericht, obwohl der erste Verfasser als Vertreter des General-Prokurators am Großen Rat tätig war. MonBAllyU, De Style, hat nachgewiesen, dass das Werk, das unter dem Namen L. van den Bryaerde veröffentlicht wurde, dem Anwalt am Rat von Flandern Fransoys de Rycke zuzuschreiben ist. ${ }^{33}$ Ordnung Style et manière de procéder (8. 8. 1559), TERLINDEN, BOLSÉE, Recueil des ordonnances II/7, 461492, Des matieres d'appel et dépendences, Art. 2.
} 
über verfahrensrechtliche Streitigkeiten argumentiert wurde.

\subsection{Historische Quellen:}

\section{Archivbestände (bes. des Archivs des Großen Rates) ${ }^{34}$}

Das heutige Archiv des Großen Rates (im Allgemeinen Reichsarchiv in Brüssel) enthält einerseits Akten und Register, die durch die damaligen Ratsbehörden selbst für die v.a. interne Benützung angefertigt wurden, und zum eigentlichen Kernarchiv des Justizhofes gerechnet werden. Andererseits gehören zum heutigen Archiv auch Dokumente, die außerhalb der Gerichtsverwaltung entstanden, bei dieser (nämlich der Hofkanzlei) abgegeben wurden und dort ständig aufbewahrt blieben (z.B. der Inhalt der Tausenden Prozess-„,Säcke“ mit Prozessstücken, Beweismaterial, Schriftsätzen usw., die durch die Parteien oder ihre Prokuratoren eingereicht und nach Prozessende nicht wieder abgeholt wurden).

\subsubsection{Das Ratsarchiv im engeren Sinne}

Aus dem Ratsarchiv im engeren Sinne können die Register (gebundene Bände), insbesondere der sog. ausführlichen Entscheidungen (sentences étendues), manchmal detaillierte Auskünfte über den Verfahrensverlauf enthalten. Die Entscheidungen wurden zwar nicht begründet; ${ }^{35}$ sie greifen auf die wichtigsten (nicht-juristischen) Argumente der Parteien zurück und bieten eine knappe Wiedergabe des Prozessganges, ggf. auch der "Zwischenfälle“ (incidents). Durch eine systematische Untersuchung der Entscheidungen könnte man den stylus curiae des Rates aber wahrscheinlich rekonstruieren. Eine solche rechtshistorische Rekonstruktion wurde für die erste Hälfte des 16. Jahrhun-

\footnotetext{
${ }^{34}$ OOSTERBOSCH, Appelprocedure.

35 WIJfFELS, Grand Conseil de Malines: La rédaction. Siehe auch: Ordonnance touchant l'extension des Sentences de ce Conseil (16. 5. 1671), Ordonnances 299-301.
}

derts für erstinstanzliche Prozesse erarbeitet. ${ }^{36}$ Die Entscheidungsregister wurden von 1470 bis ca. 1585 verzeichnet und enthalten für diese Periode etwa 10.000 Entscheidungen.

\subsubsection{Das Ratsarchiv im modernen Sinne}

Aus dem Ratsarchiv im modernen Sinne sind die Prozessakten der Parteien (dossiers) von Bedeutung. Die Prozessakten sind teilweise nach Instanzen aufgeteilt, teilweise nach ihrer territorialen Herkunft. So gibt es eine Reihe „Prozesse in erster Instanz", die theoretisch alle erhaltenen Akten erstinstanzlicher Prozesse enthält, ungeachtet ihrer territorialen Herkunft. Zwei Reihen enthalten Prozessakten in Rechtssachen, die sich auf die Interessen großer adliger Familien beziehen (obwohl solche Rechtsstreitigkeiten auch in den anderen Reihen vorkommen). Die meisten übrigen Reihen sind sog. Appellationen aus einer bestimmten Provinz, deren Provinzialrat dem Großen Rat untergeordnet war: so gibt es die Reihe "Appellationen aus Flandern“, „Appellationen aus Artois", "Appellationen aus Holland", "Appellationen aus Namur", "Appellationen aus Luxemburg" usw. Diese Reihen sind nur zum Teil verzeichnet; nur für die etwa 1.000 Akten aus den Grafschaften Holland und Seeland kann man von einem wohl fast vollständigen Verzeichnis ausgehen. ${ }^{37}$ Die Bezeichnung „Appellationen“ trifft im übrigen nur teilweise $\mathrm{zu}$, weil sich in diesen Akten auch manche Prozesse finden, die aufgrund eines anderen Rechtsmittels - Reformationen, Revisionen u.a. behandelt wurden, wobei ein als "Appellation"

\footnotetext{
${ }^{36}$ Siehe die oben schon genannte Dissertation von VAN RHEE, Litigation and Legislation.

${ }^{37}$ Siehe meine oben schon erwähnte Rezension: WiJFFELS, Dutch litigation. Für kleinere Reihen von Appellationsakten aus einem bestimmten Territorium gibt es außerdem Kurzverzeichnisse. Für Teile der weitaus umfangreichsten Reihe des Archivs des Großen Rates, die die sog. Appellationen aus Flandern enthält, ist ein zuverlässiges Findbuch vorhanden: OOSTERBOSCH, Grote Raad.
} 
klassifizierter Prozess bisweilen eher wie ein erstinstanzliches Verfahren verlief. Die Einstufung vieler Prozessakten als "Appellationen" betont immerhin, dass die große Mehrzahl von Rechtssachen, die vor dem Großen Rat eingebracht wurden, wohl Appellationssachen im engeren Sinne waren, und dass der Große Rat deshalb auch allgemein hauptsächlich als Appellationsgericht angesehen wurde.

Zum Schluss dieser allgemeinen Beobachtungen über die Quellen des Appellationsverfahrens ist noch zu betonen, dass auch der Gegensatz zwischen Normativität und Realität der Verfahrenspraxis nicht absolut war, und sei es nur, weil die Normativität die Möglichkeit von Ausnahmen vom „Musterverfahren“ immer vorsah oder einkalkulierte. Ein charakteristisches Beispiel aus der Alltagspraxis ist die recht häufige Anwendung der requête-civile, die am Großen Rat, anders als in Frankreich, ihre ursprüngliche Bedeutung als ein Rechtsmittel, das im Laufe des Verfahrens (also als ein incident oder "Zwischenfall“) eingelegt wurde, behielt, öfter, um eine vom ordentlichen Verfahren abweichende Sondererlaubnis zu bekommen. ${ }^{38}$ Im Laufe eines Appellationsverfahrens konnte eine Partei durch eine requête-civile nicht nur technische Änderungen beantragen (z.B. im Hinblick auf eine Fristverlängerung), sondern auch solche, die es ihr ermöglichten, den ordentlichen Prozessverlauf wesentlich zu umgehen (z.B. um im Appellationsverfahren neue Tatsachen vorbringen $\mathrm{zu}$ können). ${ }^{39}$

\footnotetext{
38 Verordnung vom April 1522, LAURENT, LAMEERE, Recueil des ordonnances II/2, 186; Ordnung Style et manière de procéder (8. 8. 1559), ebd. II/7, 461-492, IX.14.

${ }^{39}$ WIJFFELS, Qui millies allegatur 1, 158-159, 2, Sachverzeichnis, s.v. "Appel“; ebd. im Verzeichnis der Allegationen die wichtigsten Rubriken De appellationibus (u.ä.) im Corpus iuris civilis (D. 49.1-13, C. 7.6269) und im Corpus iuris canonici (X. 2.28, Sextus 2.15, Clem. 2.12).
}

\section{Appellationsverfahren (I): Das eigentliche Appellationsverfahren ${ }^{40}$}

Das eigentliche Appellationsverfahren am Großen Rat, wenigstens zur Zeit seiner Entstehung als Appellationsgericht, setzte voraus, dass der vorherige Prozess, dessen Entscheidung in Berufung angefochten wurde, durch den Großen Rat als sog. proces par escript (proces bij geschrifte, schriftliches Verfahren) bezeichnet wurde, und dementsprechend ohne neue Beweisführung oder Untersuchungsmaßnahmen überprüft werden konnte. Ein solches Appellationsverfahren schloss deshalb auch die Berücksichtigung neuer Tatbestände aus. Begriff und Ausdruck des proces par escript waren wohl der französischen Praxis des Pariser Parlement entnommen; am Großen Rat wurde als Voraussetzung angesehen, dass aus der vorhergehenden Prozessinstanz mindestens die richterliche Bestimmung, die den Prozess ratione materiae und ratione personae festlegte (appointement dispositif), und der formelle Antrag der Parteien auf Entscheidung in schriftlicher Form vorhanden waren. ${ }^{41}$ In einem allgemeineren Sinne setzte die Anerkennung des vorangegangenen Verfahrens als

\footnotetext{
${ }^{40}$ Für eine kurze Rekonstruktion eines Appellationsverfahrens aus den Jahren 1544-1545 siehe WIJFFELS, Qui millies allegatur 1, 32-45; die Prozessakten (Brüssel, Algemeen Rijksarchief, Grote Raad van Mechelen, Beroepen uit Holland Nr. 446, siehe HuUsseN, Inventaris) enthalten viele Stücke, die das Appellationsverfahren dokumentieren, z.B. eine Kopie des Befehls, wonach das Appellationsverfahren am fürstlichen Hof geführt werden konnte, und die Relation des Gerichtsdieners (huissier), der mit der Exekution dieser Anordnung beauftragt war (Dok. b-I und b-II); in diesem Verfahren haben auch die Beklagten gegen den Kläger appelliert, die Relation der Vollstreckung ihrer Appellation findet sich in den Prozessakten (Dok. c). Weiter enthalten die Unterlagen auch Akten, die "Zwischenfälle“ (incidents) im Laufe des Appellationsverfahren dokumentieren (Dok. g, h, u.a. über Anträge wegen Fristverlängerung).

${ }^{41}$ VAN RHEE, Litigation and Legislation 128-129.
} 
proces par escript voraus, dass die wichtigsten Stufen des Vorverfahrens schriftlich durchgeführt worden waren, und der Große Rat aufgrund dieser Unterlagen die Entscheidung des vorigen Gerichts überprüfen konnte. Wenn das vorhergehende Verfahren vor einem fürstlichen Provinzialrat stattgefunden hatte, war dies immer ein schriftlicher Prozess. Bei den „unteren“ Gerichten (diese Bezeichnung trifft freilich für die Frühzeit des Rates nicht ganz zu, denn es handelte sich meistens um die traditionellen ordentlichen Gerichte, die bis zur Entwicklung der fürstlichen Justizräte ihre gerichtliche Tätigkeit in erster und letzter Instanz ausgeübt hatten) blieb die Lage lange unterschiedlich: Im 15. Jahrhundert entsprach das Verfahren vor diesen Gerichten nur in Einzelfällen der Voraussetzung der Schriftlichkeit. Dieser Unterschied hatte große Folgen für den weiteren Verlauf des Appellationsverfahrens: Gegen eine Entscheidung in einem Prozess, der nicht als proces par escript anerkannt wurde, konnte zwar Berufung eingelegt werden, der Appellationsprozess folgte dann aber am Großen Rat dem Verfahrensmodell in erster Instanz, so dass die Tatbestände von neuem ermittelt und bewiesen werden mussten. $^{42}$

War der vorhergehende Prozess als schriftliches Verfahren anerkannt, sollte der Große Rat prüfen, so die damals gebräuchliche Formel, ex eisdem actis, an bene vel male iudicatum sit (aufgrund derselben Akten, ob gut oder schlecht geurteilt worden sei). Diese grundsätzliche Beschränkung des Appellationsprozesses wird bisweilen als Nachwirken der älteren Tradition erklärt, als die Appellation in den Niederlanden noch nicht bekannt war und eine Entscheidung durch eine Art Urteilsschelte gegen die Richter angefochten werden konnte; ${ }^{43}$ vielleicht kann man diese Auffassung auch in der noch bis weit ins 16. Jahr-

\footnotetext{
42 VAN Rompaey, De Grote Raad 451.

${ }^{43}$ GANSHOF, Etude.
}

hundert nachzuweisenden Praxis spüren, die Richter des angefochtenen Urteils im Appellationsverfahren als "Appellierte“ (appelés, geappeleerden) zu bezeichnen (gegenüber dem intimé, geïntimeerde, d.h. dem Berufungsbeklagten). Das Einlegen der Berufung war zweistufig: Erstens musste die Berufung eingelegt werden, und zwar entweder unmittelbar nachdem das angegriffene Urteil ergangen war oder innerhalb von zehn Tagen, zweitens musste der Berufungskläger seine Appellation innerhalb von drei Monaten "erheben“ (relever, verheffen), also verfolgen. ${ }^{44}$ Dies geschah, indem der Berufungskläger eine Ladungsschrift des Großen Rates (lettres patentes de relèvement d'appel) zustellen ließ. Die Ladungsschrift enthielt im 15. Jahrhundert eine Anordnung an die Richter des angefochtenen Urteils, ihre Entscheidung zu begründen und dem Großen Rat die Prozessakten zu übersenden, ${ }^{45}$ und eine Anzeige an den Berufungsbeklagten, im Appellationsverfahren zu erscheinen. 46

Wegen dieser Zweistufigkeit der Klageerhebung in Berufung war es möglich, dass der Berufungskläger die Appellation zwar einlegte, sie später aber nicht „erhob“. Nach drei Monaten wurde die Appellation dann als "verlassen“ betrachtet. In der Zwischenzeit konnte die Gegenpartei auch selbst die Initiative ergreifen und die Appellation anstelle des Berufungsklägers "erheben", was in der damaligen Verfahrens-

${ }^{44}$ Verordnung vom April 1522, LAURENT, LAMEERE, Recueil des ordonnances II/2, 186-187; Ordnung Style et manière de procéder vom 8. 8. 1559, ebd. II/7, 461-492, XIII.2.

${ }^{45}$ Fristregelung in der Ordnung Style et manière de procéder vom 8. 8. 1559, ebd. XIII.7-8.

${ }^{46}$ Siehe im Formularbuch Leiden, Universiteitsbibliotheek, BPL 54, fol. $143^{\mathrm{v}}-145^{\mathrm{r}}$, das Formular eines Relief d'appel avec les clauses d'inhibicion et deffences, mit Marginalien über einige Beschränkungen des Appellationsrechts, die wesentlich den Paragraphen V.10-15 der Ordnung Style et manière de procéder vom 8. 8. 1559, TERLINDEN, BOLSÉE, Recueil des ordonnances II/7, 461-492, entsprechen. 
terminologie „antizipieren“ (anticiper, also vorgreifen) hieß. ${ }^{47}$ Der Berufungsbeklagte wurde dann an die Stelle des Klägers gesetzt, als „Antragsteller einer Antizipation" (impétrant en matière d'anticipation), und der ursprüngliche Berufungskläger an die Stelle des Beklagten (anticipé) gegenüber seiner Gegenpartei.48 Ziel der Antizipation war entweder, die Appellation für nicht mehr zulässig erklären zu lassen, oder den Berufungskläger zu zwingen, seine Gründe für die Appellation zu erläutern, damit der Appellationsprozess weitergeführt werden konnte. ${ }^{49}$

Auch das Antizipationsverfahren konnte gelegentlich zu "Zwischenfällen" (incidents) führen. Als Beispiel kann ein Fall aus dem Jahre 1570 dienen, in dem der Antragsteller ein Prokurator am Großen Rat (also ein professioneller Rechtspraktiker) war. Seine Gegner hatten gegen eine Entscheidung der Verwalter des Waisenhauses in Mecheln an das Mechelner Stadtgericht appelliert, und gegen das Urteil des Stadtgerichts anschließend an den Großen Rat. ${ }^{50}$ Der Prokurator als Berufungsbeklagter stellte einen Antrag, um die Appellation, die noch nicht "erhoben" war, zu "antizipieren". Nachdem den Berufungsklägern mehrfach Termine gesetzt worden waren, und ihre Säumnis auch formell durch den Rat festgestellt worden war, antworteten sie schließlich, dass sie nicht eine Appellation, sondern eine Reformation eingelegt hätten, für die längere

\footnotetext{
${ }^{47}$ Verordnung vom April 1522, LAURENT, LAMEERE, Recueil des ordonnances II/2, 186; Ordnung Style et manière de procéder vom 8. 8. 1559, ebd. II/7, 461-492, XIII.1.

${ }^{48}$ Leiden, Universiteitsbibliotheek, BPL 54, fol. 158 ${ }^{\mathrm{v}}$ $149^{\mathrm{r}}$ (mit Marginalien).

${ }^{49}$ Die Decisiones-Sammlung von Paulus Christinaeus (Christinaeus, Practicarum quaestionum [...] Decisiones) bezeugt, dass für die Praxis Rechtsfragen mit Bezug auf den einseitigen oder vereinbarten Verzicht auf eine (schon eingelegte) Appellation allerdings relevant waren.

${ }^{50}$ Für das kleine Territorium von Mecheln und einige Dörfer der Umgebung gab es kein eigenes Appellationsgericht, so dass der Große Rat selbst als (territoriales) Appellationsgericht zuständig war (ohne die Möglichkeit zu einer weiteren Appellation am selben Justizhof).
}

Fristen galten; in einem Zwischenverfahren vor einem Ratskommissär behaupteten sie außerdem, dass gegen die betreffenden Urteile des Mechelner Stadtgerichts nur Reformation, aber keine Appellation eingelegt werden dürfe. Der Rat lehnte den Antrag des Prokurators $a b$, legte aber zugleich der Gegenpartei die Bezahlung der Kosten dieses "Zwischenfalls" auf. (Der weitere Verlauf des Prozesses konnte in den Urteilsregistern der 1570er Jahren nicht ermittelt werden. $)^{51}$

Die Regelung der Säumnis war für Appellationsprozesse strenger als für Verfahren in erster Instanz,52 auch wenn die Praxis sich manchmal als milder erwies. Der Berufungskläger, der "Antizipant-Beklagte“ oder der "AntizipierteKläger", der den ersten Termin versäumte, konnte in der Theorie bereits abgewiesen und in die Kosten der anderen Partei und zu einer Strafzahlung wegen der „unbesonnenen Appellation" (fol appel) verurteilt werden. Der Berufungsbeklagte sollte erst nach der zweiten Säumnis bestraft werden.

Das Erscheinen vor Gericht geschah meistens durch Prokuratoren, wobei es üblich war, dass die "Appellierten" und Berufungsbeklagten durch denselben Prokurator vertreten wurden. In den (mündlichen) Plädoyers legte der Berufungskläger die Argumente für seine Appellation dar, um nachzuweisen, dass das angefochtene Urteil nicht gegründet sei; der Berufungsbeklagte und die "Appellierten" behaupteten dagegen, dass das Urteil gegründet sei und die Appellation abzuweisen sei. Diese Auseinandersetzung konnte, wenn nötig, in weiteren Plä-

\footnotetext{
${ }^{51}$ Brüssel, Algemeen Rijksarchief, Grote Raad van Mechelen, Geëxtendeerde Sententiën 870.96 (14351440). DE SMIDT, Chronologische Lijsten Nr. 3870, 251.

52 VAN ROMPAEY, De Grote Raad 455. Für das 16. Jahrhundert und später siehe die Verordnung vom April 1522, LAURENT, LAMEERE, Recueil des ordonnances II/2, 183 (Säumnis des Klägers) und 184 (Säumnis des Beklagten); Ordnung Style et manière de procéder vom 8. 8. 1559, ebd. II/7, 461-492, XIII.10-12 (Säumnis des Klägers), 13-14 (Säumnis des Beklagten), 15-16 (Säumnis im Fall einer Antizipation).
} 
doyers (Replik, Duplik usw.) fortgeführt werden. ${ }^{53}$

Am Ende dieser Plädoyers erließ der Rat eine Verfügung - appointement -, um die Schlussanträge zu bestätigen, wobei er den Parteien erlaubte, Schriftsätze oder ein motif de droit (womit meistens eine stärker juristisch, d.h. gelehrt-rechtlich geprägte Denkschrift, eine Art Fachgutachten, gemeint war ${ }^{54}$ ) einzureichen.

Weil in diesem Appellationsverfahren im engeren Sinne keine Ermittlungen oder eine Beweisführung stattfanden, konnte der Rat dann ohne weitere Schritte zur Überprüfung der Rechtssache (der sog. visitation) übergehen.

Die Vorbereitung dieser Überprüfung wurde wie in den meisten Hauptverfahren - einem Rat anvertraut, der als Berichterstatter auftrat. In einer Beratung des Richterkollegiums erläuterte er seine Relation ${ }^{55}$ und seinen Vorschlag für das Urteil.

Wenn das Urteil die Appellation abwies (was nach den bisher verfügbaren Daten meistens der Fall gewesen sein dürfte), konnte der Berufungskläger zusätzlich noch in die Kosten des Berufungsverfahrens sowie zu einer Strafzahlung wegen fol appel (im 15. Jahrhundert $60 £$ von 40 g.) verurteilt werden. ${ }^{56}$ Der Große Rat

\footnotetext{
${ }^{53} \mathrm{Im}$ Rahmen dieses knappen Beitrages können nur die wichtigsten Merkmale der Appellation erwähnt werden. Die damalige Rechtsprechungs- und Konsilienliteratur weist auf viele andere Besonderheiten des Appellationsverfahrens hin. Siehe auch TEN RAA, Appèl.

${ }^{54}$ WIJFFELS, Qui millies allegatur 1, 71-73.

${ }^{55}$ Diese Relationen wurden, so weit ersichtlich, nicht aufbewahrt. Darüber hinaus sind für den Großen Rat nur wenige richterliche Aufzeichnungen oder Sammlungen bekannt, in denen das Vorgehen bei der Besprechung einer Relation dargestellt wird, dazu WiJFFELS, Legal Records and Reports.

${ }^{56}$ VAN ROMPAEY, De Grote Raad 460-464. In der Praxis wurde manchmal über die Verhängung dieser Strafzahlung in besonderen Umständen gestritten, siehe z.B. SAINCT VAAST, DU LAURY, La jurisprudence des Pais-Bas autrichiens, Arrêt 33, 54-55: „En une
}

verfügte in dieser Frage jedoch über einen Ermessensspielraum und konnte sowohl im Hinblick auf die Kosten wie hinsichtlich der Strafzahlung, z.B. aus Billigkeitserwägungen, eine abweichende Entscheidung treffen. Wie in erstinstanzlichen Prozessen, wurden auch in Appellationsverfahren die Kosten öfter zwischen Kläger und Beklagten aufgeteilt. ${ }^{57}$ Die Entscheidung der Richter stützte sich jedenfalls nicht notwendigerweise auf die wirtschaftliche Lage der Parteien: Wie mehrere Fälle bezeugen, wurde auch versucht, Strafzahlungen und Kosten selbst von unvermögenden Parteien einzutreiben.

Wenn der Große Rat die Appellation für zulässig und gegründet hielt, musste das Urteil (ggf. die aufeinander folgenden Urteile der unteren Gerichte) aufgehoben und abgeändert werden. Diese Entscheidung findet man ganz am Ende der Urteile des Großen Rates in einem meist kurzen, formelhaften und nur stereotyp begründeten Tenor. In der Anfangszeit des Rates konnte ein Schöffengericht, dessen Urteil auf diese Weise für ungegründet erklärt wurde, zu einer Strafzahlung verurteilt werden; den Richtern der fürstlichen Provinzialräte dagegen wurde eine solche Strafzahlung nicht auferlegt. ${ }^{58}$ Das Appellationsurteil des Großen Rates konnte die angefochtene Entscheidung auch teilweise aufheben und abändern, teilweise bestätigen. In einem solchen Fall konnte die Strafzahlung verringert und die Prozesskosten konnten aufgeteilt werden.

Im Fall der Evokation eines Prozesses, der schon das Stadium eines Appellationsverfahrens erreicht hatte, wurde die Sache ebenfalls als Appellation behandelt. Bei der Festsetzung der Strafzahlung und der Kosten wurde aber

cause d'Apel évoquée au Grand Conseil, l'Apellant étant declaré Fol-Apellant, ne doit être condamné en l'amende de frivol Apel, selon le taux dudit Grand Conseil, mais selon celui des Juges, desquels la cause a été évoquée".

${ }^{57}$ VAN RHEE, Litigation and Legislation 218-222.

${ }^{58}$ VAN Rompaey, De Grote Raad 460-461. 
manchmal berücksichtigt, dass der Berufungskläger nicht von sich aus die Initiative für eine Appellation beim Großen Rat ergriffen hatte, und deshalb auch nicht unbedingt für Strafzahlung und Kosten, die normalerweise anlässlich eines solchen Berufungsverfahrens fällig wurden, haften sollte.

Bei der Vollstreckung eines Urteils des Großen Rates (in Appellationsprozessen und anderen Verfahren) $)^{59}$ konnten noch viele "Zwischenfälle“ (incidents) auftreten, zu denen die Register und Akten des Rates auch regelmäßig Material bereitstellen. ${ }^{60}$

\section{Appellationsverfahren (II): Alternative zum Prozess ex eisdem actis}

Die Prozessschriften, die durch die Parteien am Großen Rat in Appellationssachen eingereicht wurden, bezeugen, dass der Rat sich in vielen Verfahren nicht auf eine Überprüfung aufgrund der Akten der vorhergehenden Instanz beschränkte. Nicht in allen Appellationsverfahren wurde entschieden, das Verfahren der Vorinstanz als proces par escript $\mathrm{zu}$ betrachten und deshalb zur Grundlage des Verfahrens in der Berufungsinstanz zu machen. Im Archiv des Großen Rates spürt man noch heute in manchen Akten eines Appellationsprozesses eine Vierteilung, die wohl mit der damaligen Verteilung der Stücke auf vier separate Prozess-,,Säcke“" übereinstimmt: einer für die Stücke, die durch den Kläger vor dem Provinzialrat eingereicht wurden, ein zweiter für die Eingaben des Beklagten in derselben Instanz, und ein dritter und

\footnotetext{
${ }^{59}$ VAN RHEE, Litigation and Legislation 261-288.

${ }^{60}$ Selbst die Gesetzgebung enthielt eine kurze Regelung über "Appellationen" gegen eine Exekution: Ordnung Style et manière de procéder vom 8. 8. 1559, TERLINDEN, BOlsÉE, Recueil des ordonnances II/7, 461492, XXI.10.
}

vierter "Sack" für die Stücke, die der Berufungskläger bzw. der Berufungsbeklagte in der Appellationsinstanz vor dem Großen Rat einbrachten.

Aber auch wenn die Akten der Vorinstanz als Grundlage anerkannt wurden, gab es mehrere Möglichkeiten, um trotzdem durch ein Ermittlungs- und Beweisverfahren neue Tatsachen vorzubringen. Die Technik der requête-civile wurde schon erwähnt. Eine andere Technik war die Überführung eines Appellationsverfahrens in ein Reformationsverfahren. ${ }^{61}$ Die Reformation war ein Rechtsmittel, das nur gegen das Urteil eines unteren Gerichts (meistens eines Schöffengerichts) eingelegt werden konnte, und zwar entweder bei einem (fürstlichen) Provinzialhof oder beim Großen Rat. Eine weitere, zweite Reformation in demselben Prozess war ausgeschlossen: Hatte eine Partei sich für eine Appellation gegen das Urteil eines Schöffengerichts entschieden, dann wurde, wie schon erwähnt, diese Appellation (bis auf einige Ausnahmen) beim Provinzialrat verhandelt; eine weitere (zweite) Appellation beim Großen Rat war ggf. nachher noch möglich. Hatte eine Partei dagegen das Rechtsmittel der Reformation gewählt, dann konnte dieses Reformationsverfahren entweder beim Provinzialrat, oder unmittelbar beim Großen Rat durchgeführt werden; im ersten Fall war es aber nicht mehr möglich, gegen die Entscheidung des Provinzialrates noch eine Reformation (oder eine Appellation) beim Großen Rat einzulegen. Im Laufe des Reformationsverfahrens wurde die Streitigkeit wie in einem erstinstanzlichen Verfahren verhandelt, also nicht ex eisdem actis. Deshalb bedeutete die Überführung einer Appellation in eine Reformation durch den Großen Rat, dass der Prozess wie von

\footnotetext{
${ }^{61}$ Dass diese Möglichkeit in der Praxis als wichtig angesehen wurde, wird schon allein durch ein spezifisches Formular bezeugt: Formularbuch Leiden, Universiteitsbibliotheek, BPL 54, fol. 146 $6^{\mathrm{r}-\mathrm{v}}$ : Appellation mue en reformation.
} 
Neuem geführt wurde. Andere Unterschiede waren, dass die Frist, innerhalb deren die Reformation eingeleitet werden musste, ein Jahr und einen Tag betrug (gegenüber der viel kürzeren Frist von 10 Tagen und darauf folgend von drei Monaten für die „Erhebung“ im Fall der Appellation); weiter, dass aus der Reformation, im Gegensatz zur Appellation, kein Suspensiveffekt erwuchs; und darüber hinaus, dass die Strafzahlung, die im Fall einer Ablehnung der Appellation dem Kläger auferlegt wurde, im Fall einer Reformation nicht verhängt wurde (obwohl sich in der Praxis doch eine entsprechende Sanktion entwickelte). Für das 15. Jahrhundert kann man feststellen, dass sich der Widerstand vieler Stadtverwaltungen gegen den Aufstieg der Appellation auch gegen das Reformationsverfahren richtete, denn in beiden Fällen wurde die Zuständigkeit der Stadtgerichte in letzter Instanz untergraben. ${ }^{62}$ Einige Städte (wie schon in der burgundischen Zeit Mecheln und Middelburg) erhielten auf eigenen Antrag Privilegien, die die Reformation gegen Urteile

${ }^{62}$ An dieser Stelle ist daran zu erinnern, dass die in diesem Aufsatz beschriebenen Merkmale der Appellation hauptsächlich Zivilprozesse betreffen. In einigen Territorien der Niederlande blieb die Appellation in Strafsachen eher eine Ausnahme: MONBALlyU, Van appellatiën ende reformatiën 264-266 (für die Grafschaft Flandern, aber mit Hinweisen auf die Lage in anderen Provinzen der alten Niederlande); DERS., Zes eeuwen strafrecht 363. MAES, Vijf eeuwen stedelijk strafrecht 148-149, erwähnt kurz denselben Sachverhalt aus der Perspektive des Mechelner Stadtrechts und einiger allgemeiner Rechtsquellen in den (südlichen) Niederlanden. Die Appellationen an den Großen Rat in Strafverfahren waren während der Neuzeit stets mit Auseinandersetzungen verbunden, insbes. mit dem Rat von Flandern, siehe z.B. GOOVAERTS, Inventaires 1, Nr. 469, 1019; 2, Nr. 1403, 1432, 1610, 1628 (eine „vorläufige” Ordnung, erlassen durch den Geheimen Rat am 30.12. 1661), 1667, 1827 (woraus sich ergibt, dass die Ordnung von 16611694 noch immer gültig war), 2147. Im Verhältnis zum Rat in Namur: ebd. 1, Nr. 1117; 2, Nr. 2273, 2274, 2291. Im Verhältnis zum Rat in Luxemburg: ebd. 2, Nr. 1895, 1979, 2026, 2369. ihrer Gerichte regelten und gewissermaßen einschränkten. ${ }^{63}$

Ein Beispiel aus den Jahren 1556-1572 illustriert, welche Verfahrensstrategien den Prozessparteien (und ihren Rechtsberatern) in der Wahl ihrer Rechtsmittel zur Verfügung stehen konnten. Ein Arzt aus Middelburg vertrat die Auffassung, er könne aufgrund einer Schuldumwandlung (Novation) für eine bestimmte Schuld nicht mehr als Bürge in Anspruch genommen werden; das Stadtgericht ordnete in einem Zwischenurteil an, er solle sich trotzdem zum Sachverhalt einlassen. Daraufhin appellierte er an den Provinzialrat von Holland, erhielt aber kurze Zeit später vom Großen Rat die Genehmigung, das Zwischenurteil von Middelburg in weiterer Folge mittels einer Reformation anzugreifen. Die Gegenpartei hatte jedoch die Appellation schon antizipiert. Der Arzt erwiderte, kraft eines Middelburger Privilegs habe er die Wahl zwischen der Appellation am Hof von Holland und der am Großen Rat; dieses Recht könne ihm (aufgrund des Grundsatzes in C. 3.1.10) nicht genommen werden. Trotzdem setzte der Arzt den Rechtsstreit später vor dem Rat in Holland als Appellant fort; der Hof entschied 1564, dass die Berufung gegen das Urteil von Middelburg ungegründet war. Der Arzt legte eine weitere Berufung beim Großen Rat ein und versuchte, durch eine requête-civile neue Tatsachen vorzubringen, während die Gegenpartei darauf bestand, dass der Prozess als "schriftliches Verfahren" betrachtet werden sollte. Der Große Rat wies die Berufung 1572 ab und entschied am selben Tag in einem separaten Urteil, dass auch der Einspruch des Arztes gegen die Pro-

\footnotetext{
${ }^{63}$ VAn RompaeY, De Grote Raad 351-352. In gewissen Fällen kann man sogar von einem Privileg de non appellando sprechen, siehe z.B. einen Rechtsstreit, in dem das Mechelner Stadtgericht eine Entscheidung der Wollweberzunft bestätigt hatte und die verurteilte Partei versuchte, am Großen Rat zu appellieren; der Einspruch gegen diese Appellation wurde durch den Großen Rat bestätigt, der Appellant, der gegen die einschlägigen Mechelner Privilegien verstoßen hatte, zu einer Strafzahlung in Höhe von 60 Gulden verurteilt (Brüssel, Algemeen Rijksarchief, Grote Raad van Mechelen, Geëxtendeerde Sententiën 871.80 (15331542); DE SMIDT, Chronologische Lijsten Nr. 3955, 279).
} 
zesskostenberechnung durch den Hof von Holland nicht begründet war. ${ }^{64}$

\section{Schlussbetrachtung: Wechselwirkung zwischen normativen Rechtsquellen, Praxis und juristischem Schrifttum}

Aus dieser allgemeinen (und unvollständigen) Skizze der Zuständigkeit und des Verfahrens in Appellationssachen am Großen Rat von Mecheln ergibt sich, dass der Rat, trotz Hinweisen auf einen eigenen stylus, dem normativen Rahmen der meisten frühneuzeitlichen Appellationshöfe, die eine gemeinrechtliche Tradition mit einem wachsenden Partikularrecht kombinierten, entsprach. Diese Tradition war geprägt durch eine Wechselwirkung zwischen Gesetzgebung, Praxis und dem (v.a. gelehrt-rechtlichen) Schrifttum. Ausdrücklich oder implizit wiesen Gesetzgebung, Praxis und Schrifttum aufeinander hin. Diese dreifache wechselseitige Beeinflussung von Rechtsquellen war schon im Spätmittelalter ein Merkmal des römischkanonischen Verfahrens gewesen, wobei die Bedeutung des Beitrags des Partikularrechts freilich je nach Gericht und seiner Zusammensetzung unterschiedlich war.

Während der Frühphase des Großen Rates im 15. Jahrhundert und weitgehend noch bis in die ersten Dezennien des 17. Jahrhunderts wurde die Praxis am Rat grundlegend geprägt durch Richter, Personal und Anwälte, die vor allem durch die gelehrte gemeinrechtliche Kultur beeinflusst waren. Später scheint sich die Praxis am Großen Rat kaum noch in neue Richtungen

\footnotetext{
${ }^{64}$ Brüssel, Algemeen Rijksarchief, Grote Raad van Mechelen, Geëxtendeerde Sententiën 872.84-85 (15171545) (1. 2. 1572 st.n.) sowie die Prozessakten, ebd., Beroepen uit Holland Nr. 525.
}

entwickelt zu haben. ${ }^{65}$ Diese Faktoren können teilweise erklären, warum das Verfahren trotz eines partikularen stylus eher zu den konventionellen gemeinrechtlichten Verfahrenstypen gerechnet werden kann. Die Berufung auf das gelehrte Recht durch sowohl die Praktiker in Einzelfällen wie den Gesetzgeber hat das Verfahren am Großen Rat dauerhaft in der gemeinrechtlichten Tradition und Kultur verankert.

\section{Korrespondenz:}

Prof. Dr. Dr. Dr. Alain Wijffels

Université catholique de Louvain

Faculté de droit et de criminologie

Institut pour la recherche interdisciplinaire en sciences juridiques

Place Montesquieu 2, 1348 Louvain-la-Neuve, Belgien Alain.Wijffels@uclouvain.be

\section{Abkürzungen: \\ $£ \quad$ Pfund \\ c. contra \\ Dok. Dokument \\ g. groten \\ Jg. Jahrgang \\ Ms. Manuscrit \\ st.n. stilus novus}

Siehe auch das allgemeine Abkürzungsverzeichnis: http://www.rechtsgeschichte.at/files/abk.pdf

\footnotetext{
${ }^{65}$ Das ist jedenfalls der erste Eindruck, der sich aus den gedruckten Rechtsprechungssammlungen des 18. Jahrhunderts ergibt. Selbst am Ende des Ancien Régime wurden noch Sammlungen vom Ende des 16. und dem Anfang des 17. Jahrhunderts veröffentlicht, siehe WiJfFELS, Legal Records and Reports 188192 (G. de Grysperre, P. de Cuvelier, C. de Humyn, N. du Fief); siehe auch DERS., Höchstgerichtsbarkeit. Die Sammlungen des 18. Jahrhunderts von JeanAlphonse de Coloma und Jean-Baptiste Hony (COLOMA, HonY, Arrêts) enthalten wenige Abschnitte über das Appellationsrecht (siehe jedoch ebd. I, 101130, Entscheidung vom 12. 2. 1713 über die Beschränkungen der Appellation in Strafsachen nach den späteren Interpretationen der Strafprozessordnungen von 1570).
} 


\section{Literatur:}

Paulus ChristinAeus, Practicarum quaestionum rerumque in supremis Belgarum curiis actarum et observatarum Decisiones, 6 Teile (Antverpiae 1661).

Jean-Alphonse de COLOMA, Jean-Baptiste HONY, Arrêts du Grand Conseil de S.M.I. et R. séant en la ville de Malines [...], 2 Bde. (Malines 1781).

Peeter CUYPERS, Guilliam CUYPERS, Tractaet van Grondt-Proceduren, ofte Uuytwerkinge van de Actie Hypothecaire, bestaande in uytwinninge van Erffelycke goederen voor Chynsen, Renten en Lasten, volgens den Styl ende costuymen van Mechelen, met verhandelinge van het IIde deel van beleyde van den 13. Titel (Mechelen 1679).

Serge DAUCHY, Wielant, Philippe, in: Patrick ARABEYRE, Jean-Louis HALPÉRIN, Jacques KRYNEN (Hgg.), Dictionnaire historique des juristes français (XII $-X X^{e}$ siècle) (Paris 2007) 781-782.

Joost DE DAMHOUDER, Practycke in civile saecken, Seer nut, profijtelijck ende nodigh allen Schouten, Borghemeesteren Magistraten ende andere Rechteren ('s Graven-Hage 1626, ND (mit einem Aufsatz von Jos MONBALlyu und Jozef Maria DaUwE) Gent 1999).

J. Th. (Tom) DE SMIDT u.a. (Hgg.), Chronologische lijsten van de Geëxtendeerde Sententiën berustende in het archief van de Grote Raad van Mechelen, Bd. 6 (Brussel 1988).

DERs., Quelques remarques sur le Ms. BPL 54 de la Bibliothèque de l'Université de Leyde, le manuscrit PRAXIS, in: J. A. ANKUM, J. E. SPRUIT, F. B. J. WubBe (Hgg.), Satvra Roberto Feenstra sexagesimum quintum annum aetatis complenti ab alumnis collegis amicis oblata (Fribourg/Schweiz 1985) 533-544.

René DEKKERS, Bibliotheca Belgica juridica. Een biobibliographisch overzicht der rechtsgeleerdheid in de Nederlanden van de vroegste tijden af tot 1800 (= Verhandelingen van de Koninklijke Vlaamse Academie voor Wetenschappen, Letteren en Schone Kunsten van België, Klasse der Letteren Jg. 13, Nr. 14, Brussel 1951).

Nicolaus Everardus, Consilia sive Responsa (1. Aufl. Lovanii 1554, benützte Ausgabe: Francofurti 1594).

François-Louis GANSHOF, Etude sur le faussement de jugement dans le droit flamand des $\mathrm{XII}^{\mathrm{e}}$ et $\mathrm{XIII}{ }^{\mathrm{e}}$ siècles, in: Bulletin de la Commission royale des anciennes lois et ordonnances de Belgique 14 (1935) 115-140.

John GILISSEN, De Grote Raad van Mechelen. Historisch overzicht, in: Miscellanea Consilii Magni. Ter gelegenheid van twintig jaar Grote Raad van
Mechelen (= Verzamelen en bewerlen van de jurisprudentie van de Grote Raad, Nieuwe Reeks 4, Amsterdam 1980) 13-44.

Philippe GodDING, Le Conseil de Brabant sous le règne de Philippe le Bon (1430-1467) (= Mémoire de la Classe des Lettres de l'Académie Royale de Belgique III/19, Bruxelles 1999).

M. GOOVAERTS, Inventaires des archives de la Belgique publiés par ordre du Gouvernement, [1:] Inventaire des mémoriaux du Grand Conseil de Malines, 2 Bde. (Bruxelles 1901-1903).

A. H. HuUsSEN, Inventaris en beschrijving van de processtukken (dossiers) behorende tot de Beroepen uit Holland Berustende in het archief van de Grote Raad van Mechelen, Algemeen Rijksarchief Brussel, Bd. 5 (Amsterdam 1968).

Joke M. I. Koster-VAN DiJK, Alain WiJfFels, Les procédures en révision au Grand Conseil de Malines (1473-1580), in: Publication du Centre Européen d'Etudes bourguignonnes (XIVe-XVIe s.) 30 (1990) 67-97.

Ch. Laurent, J. LAmeere (Hgg.), Recueil des ordonnances des Pays-Bas, Deuxième série: 1506-1700, Bd. 2 (Brüssel 1898).

Marie-Charlotte le Bailly, Chr. M. O. Verhas, Hoge Raad van Holland, Zeeland en West-Friesland (1582-1795). De hoofdlijnen van het procederen in civiele zaken voor de Hoge Raad zowel in eerste instantie als in hoger beroep (= Procesgidsen 5, Hilversum 2006).

Louis-Theo MAES, Het Parlement en de Grote Raad van Mechelen 1473-1797 (Antwerpen-Rotterdam 2009).

DERS., Vijf eeuwen stedelijk strafrecht. Bijdrage tot de rechts- en cultuurgeschiedenis der Nederlanden (Antwerpen-'s-Gravenhage 1947).

Jos Monballyu, De Style ende maniere van procederen in de camere van den Raede van Vlaenderen (1521) van Fransoys de Rycke († 1525) ten onrechte toegeschreven aan Lambrecht van den Bryaerde, president van de Grote Raad van Mechelen, in: Tijdschrift voor Rechtsgeschiedenis 49 (1981) 361388.

DERS., ,Van appellatiën ende reformatiën': de ontwikkeling van het hoger beroep bij de Audiëntie, de ,Camere van den Rade' en de Raad van Vlaanderen (ca. 1370-ca. 1550). Bijdrage tot de ontstaansgeschiedenis van het hoger beroep in de Nederlanden, in: Tijdschrift voor Rechtsgeschiedenis 61 (1993) 237-276.

DERS., Zes eeuwen strafrecht. De geschiedenis van het Belgische strafrecht (1400-2000) (Leuven-Voorburg 2006). 
Michel OOsterbosch, Appelprocedure, dossierbehandeling en registervoering bij de Grote Raad van Mechelen, in: C. H. van Rhee, F. Stevens, E. PERSOONS (Hgg.), Voortschrijdend procesrecht. Een historische verkenning (Leuven 2001) 3-25.

DERS., Grote Raad voor de Nederlanden te Mechelen. Procesbundels Beroepen uit Vlaanderen, Ordinaris Processen (Nr. 1-1000) (Brussel 1998).

Ordonnances, statuts, stile, et maniere de proceder, faits et decretez par le Roi Don Philippe II. pour son Grand Conseil [...]. Nouvelle Edition augmentée de plusieurs Ordonnances et Reglemens jusques à l'An 1719 (Bruxelles 1721).

Pierre-Claude-Marie de SAINCT VAAST, Remi-Albert DU LAURY, La jurisprudence des Pais-Bas autrichiens établie par les arrêts du Grand Conseil de Sa Majesté Impériale et Catholique, Residant en la Ville de Malines (Brusselle 1717).

Hugo de SCHEPPER, De Geheime Raad als hoogste rechtscollege in de Nederlanden (1504-1580), in: Rob HuijBrecht, Handelingen van het Tweede Hof van Holland Symposium gehouden op 14 november 1997 in de Trêveszaal te Den Haag (= Algemeen Rijksarchief publikatiereeks 7, Den Haag 1998) 39-48.

DERS., De Grote Raad van Mechelen als hoogste rechtscollege in de Nederlanden?, in: Bijdragen en Mededelingen betreffende de Geschiedenis der Nederlanden 91 (1978) 389-411, auch [in einer leicht veränderten Fassung] in: Miscellanea Consilii Magni. Ter gelegenheid van twintig jaar Grote Raad van Mechelen (= Verzamelen en bewerlen van de jurisprudentie van de Grote Raad, Nieuwe Reeks 4, Amsterdam 1980) 171-194.

DERS., Rechter en Administratie in de Nederlanden tijdens de zestiende eeuw. Rede voor de faculteit der rechtsgeleerdheid uitgesproken in de aula van de Universiteit van Amsterdam op maandag 22 september 1980 bij de aanvaarding van de opdracht van gewoon hoogleraar in de geschiedenis van het recht, in het bijzonder de geschiedenis van de staatsinstellingen (Alphen aan de Rijn 1981).

Christiaan M. G. TEN RAA, Appèl à minima en reconventie: over de mogelijkheid van een incidentele voorziening in hoger beroep gericht tegen een vonnis in reconventie gewezen terwijl het principaal beroep beperkt was tot de uitspraak in conventie (Deventer 1972).

TERLINDEN, J. BolséE (Hgg.), Recueil des ordonnances des Pays-Bas, Deuxième série: 1506-1700, Bd. 7, (Bruxelles 1957).

Raoul C. van CAEnegem, History of European Civil Procedure (= International Encyclopedia of Com- parative Law 16: Civil Procedure, Chapter 2, Tübingen u.a. 1973).

Raoul VAN DER MADE, Formulaires du XVIe siècle, conservés aux archives communales de Huy, in: Bulletin de la Commission Royale des Anciennes Lois et Ordonnances de Belgique 20 (1962) 521530.

Willem VAN DER TANERIJEN, Boec van der loopender practijken der raidtcameren van Brabant, hg. v. Eg. I. STRUBBE (Brüssel 1952).

C. H. (Remco) VAN RHEE, Litigation and Legislation. Civil procedure at First Instance in the Great Council of the Netherlands in Malines (1522-1559) (= Studia / Archives Générales du Royaume et Archives de l'Etat dans les Provinces 66, Brussel 1997).

Jan VAn Rompaey, De Grote Raad van de Hertogen van Boergondië en het Parlement van Mechelen (=Verhandelingen van de Koninklijke Academie voor wetenschappen, Letteren en Schone Kunsten van België, Klasse der Letteren XXXV/73, Brussel 1973).

DERS., De procedure in beroep bij het Parlement van Mechelen, in: Consilium magnum 1473-1973. Herdenking van de 500 verjaardag van de oprichting van het Parlement en Grote Raad van Mechelen, Colloquium (Brussel-Mechelen) Commémoration du 500e anniversaire de la création du Parlement et Grand Conseil de Malines, Colloque (Bruxelles-Malines) 8.-9. 12. 1973 (Brussel 1977) 371-381.

Udo WAgner, Niederlande, in: Helmut CoING (Hg.), Handbuch der Quellen und Literatur der neueren europäischen Privatrechtsgeschichte, Bd. 2, Teilbd. 2 (München 1976) 1399-1430.

Johannes WAMESIUS, Responsorvm sive consiliorvm ad ivs, forvmque civile pertinentivm, centvria [prima-quinta] (1. Aufl. Lovanii 1625-1632), benützte Ausgaben: Lovanii 1625-1632 und Antwerpen 1639-1641.

DERS., Responsorum sive consiliorum de iure pontificio, Bd. [1-2] (1. Aufl. Lovanii 1605-1618), benützte Ausgabe: Lovanii 1643.

Ders., Tractatus de appellationibus. Ad Praxin forensem accommodatissimus (Lovanii 1600).

Wilhelmus George Philip Erich WEDEKIND, Bijdrage tot de kennis van de ontwikkeling van de procesgang in civiele zaken voor het Hof van Holland in de eerste helft van de zestiende eeuw (= Bijdragen van het Instituut voor Rechtsgeschiedenis van den Rijksuniversiteit Utrecht 8, Assen 1971).

Philips Wielant, Practijke civile, hg. v. Eg. I. StRUBBE (Amsterdam 1968, ND der Ausgabe Antwerpen 1573). 
Alain WiJfFels, A Consultancy on Wine Imports, in: Tijdschrift voor rechtsgeschiedenis 73 (2005) 321355.

DERS., La coutume dans les consultations de Wamèse, in: Bart CoppeIN, Fred STEVENS, Laurent WAELKENS (Hgg.), Modernisme, tradition et acculturation juridique. Actes des Journées internationales de la Société d'Histoire du Droit tenues à Louvain, 28 mai-1 juin 2008 (= Iuris Scripta Historica 27, Brussel 2011) 107-124.

DERS., Dutch litigation before the Great Council of Mechlin: An additional calendar of the ,Appeals from Holland', in: Tijdschrift voor rechtsgeschiedenis 77 (2009) 539-550.

DERS., Grand Conseil de Malines: recueil de jurisprudence de Guillaume de Grysperre. Texte intégral, in: DERS. (Hg.), Case Law in the Making. The Techniques and Methods of Judicial Records and Law Reports, Bd. 2 (= Comparative Studies in Continental and Anglo-American Legal History 17/2, Berlin 1997) 337-388.

DERS., Grand Conseil de Malines: La rédaction des sentences étendues et le recueil de jurisprudence de Guillaume de Grysperre, in: DERS. (Hg.), Case Law in the Making. The Techniques and Methods of Judicial Records and Law Reports, Bd. 1 (= Comparative Studies in Continental and AngloAmerican Legal History 17/1, Berlin 1997) 299316.

DERS., The Great Council of Malines, in: Bernhard DiESTELKAMP (Hg.), Oberste Gerichtsbarkeit und zentrale Gewalt im Europa der frühen Neuzeit (= Quellen und Forschungen zur höchsten Gerichtsbarkeit im Alten Reich 29, Köln-WeimarWien 1996) 113-125.
DERS., Grote Raad voor de Nederlanden te Mechelen (ca. 1445-1797), in: E. AERTS u.a. (Hgg.), De centrale overheidsinstellingen van de Habsburgse Nederlanden (1482-1795), Bd. 1 (= Studia / Algemeen Rijksarchief en Rijksarchief in de Provinciën 55, Bruxelles 1994) 448-461.

DERS., Höchstgerichtsbarkeit in den südlichen Niederlanden (16./17. Jahrhundert): Nicolas du Fief und die Praxis des Großen und des Geheimen Rates, in: Rolf LIEBERWIRTH, Heiner LÜCK (Hgg.), Akten des 36. Deutschen Rechtshistorikertages, Halle an der Saale, 10.-14. September 2006 (Baden-BadenBern-Stuttgart 2008) 626-647.

DERS., Ius commune and International Wine Trade. A Revision (Middelburg c. Antwerp, 1548-1559), in: Tijdschrift voor Rechtsgeschiedenis 71 (2003) 289317.

DERS., Legal Records and Reports in the Great Council of Malines (15th to 18th Centuries), in: John H. BAKER (Hg.), Judicial Records, Law Reports, and the Growth of Case Law (= Comparative Studies in Continental and Anglo-American Legal History 5, Berlin 1989) 181-206.

DERS., Qui millies allegatur. Les allégations du droit savant dans les dossiers du Grand Conseil de Malines (causes septentrionales, ca. 1460-1580), 2 Teile (= Rechtshistorische Studien 11, Leiden 1985, auch als: Verzamelen en bewerken van de jurisprudentie van de Grote Raad, Nieuwe Reeks 10, Amsterdam 1985).

DERS., Van Paul van Christijnen (†1631) tot JeanAlphonse de Coloma († 1739): rechters en advocaten bij de Grote Raad van Mechelen tegen de achtergrond van de zeventiende-eeuwse Europese rechtsontwikkeling, in: De zeventiende eeuw 9 (1993) 3-14.

\section{Zusammenfassung}

Der Große Rat entstand im 15. Jahrhundert nicht nur als ein Appellationsgericht, sondern er verfügte auch über eine vielseitige erstinstanzliche Zuständigkeit. Darüber hinaus gab es verschiedene andere Rechtsmittel, durch welche man eine Rechtssache vor den Rat bringen konnte (z. B. Opposition, Reformation, Revision). Als Appellationsgericht war der Rat grundsätzlich für die Überprüfung von Entscheidungen der Provinzialhöfe zuständig. Allerdings gab es eine Vielzahl von Ausnahmen, so dass auch Urteile unterer Gerichte (z.B. Stadtgerichte) direkt vor dem Rat angefochten werden konnten. Das Appellationsverfahren entsprach im 15. Jahrhundert im Wesentlichen dem Muster des gelehrten römischkanonischen Verfahrensrechts, wurde in der burgundischen Zeit aber auch durch die französische Praxis, insbes. die des Pariser Parlement, beeinflusst. Daneben entwickelte der Rat allmählich seinen eigenen „Stil“. Sein Verfahren wurde darüber hinaus durch die fürstliche Gesetzgebung (insbes. des späten 15. und des 16. Jahrhunderts) geregelt. Obwohl das Appellationsverfahren in der Regel die Überprüfung des angefochtenen Urteils aufgrund der Akten der Vorinstanz vorsah (ex eisdem actis, an bene vel male iudicatum sit), zeigt die Praxis, dass die Parteien, insbes. durch das Mittel der requête-civile, Abweichungen 
vom ordentlichen Prozessverlauf erreichen konnten. Auf diese Weise wurde u.a. das Vorbringen neuer Tatsachen einschließlich einer eigenen Beweisführung möglich. Damit näherte sich das Appellationsverfahren in der Praxis teilweise dem erstinstanzlichen Prozess an.

\section{Summary}

The Great Council ("Grote Raad") developed in the $15^{\text {th }}$ century not only as an appellate court but also as a first instance court, competent for a variety of cases. Moreover, other remedies were sometimes available to bring a case before the Council (opposition, reformation, revision). As an appellate court, the Council mostly heard appeals from provincial Councils, but there was a variety of exceptions, which meant that the Council could also revise sentences of some lower courts (e.g. municipal courts). In the $15^{\text {th }}$ century the Council's proceedings in appeals mostly followed the model of the Roman and Canon law. During the Burgundian period, however, they were also influenced by French practice, esp. that of the Parlement in Paris. Moreover, the Council gradually developed its own "style". Proceedings were also regulated by the sovereign's statutes (late $15^{\text {th }}$ and $16^{\text {th }}$ centuries). Appellate proceedings at the Council normally meant the revision of judicial decisions by examination only of the previous instance's records ("ex eisdem actis, an bene vel male iudicatum sit"). In practice, however, parties were able to secure exceptions from that rule, especially by making use of the so called "requête-civile". By these means they were able to introduce new facts to the proceedings and to produce additional evidence. In such cases, the Council's appellate proceedings in practice partly resembled its proceedings in the first instance. 
